\title{
Numerical simulation of the motion of a Taylor drop in a non-Newtonian fluid
}

\author{
Elham Usefi $^{1} \cdot$ Morteza Bayareh $^{1}$
}

Received: 20 March 2020 / Accepted: 28 May 2020 / Published online: 7 June 2020

(c) Springer Nature Switzerland AG 2020

\begin{abstract}
In this study, the motion of a Taylor drop in a non-Newtonian fluid in a vertical tube is investigated numerically. The governing equations are solved using a volume of fluid (VOF) method in a symmetric geometry using OpenFOAM software. The continuous phase fluid behaves as shear-thinning fluid and modeled using Bird-Carreau non-Newtonian model. The effects of the power-law index $(n)$, Froude number $(0.05 \geq \mathrm{Fr} \geq 0.31)$ and the Eötvös number $\left(9.5 \geq \mathrm{Eo}_{D} \geq 123.7\right)$ on the motion and deformation of Taylor drop are studied. The results show that the Froude number increases by increasing the power-law index, leading to an increase in the thickness of the thin layer of the fluid around the drop. For very small power-law indices, $n$ has little effect on the Froude number. The drop deformation in Newtonian fluid $(n=1)$ is less than that in non-Newtonian ones. It is revealed that the Froude number increases with the Eötvös number. As the Eötvös number decreases, the thickness of the thin layer of the fluid and the drop deformation decrease. The results demonstrate that for $\mathrm{Eo}_{D}>70$, the effect of the Eötvös number on the Froude number is negligible.
\end{abstract}

Keywords Taylor drop · Finite volume scheme · OpenFOAM software · Bird-Carreau model · Eötvös number · Froude number

\section{List of symbols}

$D \quad$ Tube diameter $(\mathrm{m})$

Eo Eötvös number

Fr Froude number

$\vec{g}$ Gravity acceleration $\left(\mathrm{m} / \mathrm{s}^{2}\right)$

$h$ The thickness of the thin layer of the fluid $(\mathrm{m})$

$L$ Taylor drop length $(\mathrm{m})$

$\vec{n} \quad$ Normal vector $(\mathrm{m})$

$p$ Pressure $(\mathrm{Pa})$

$R \quad$ Taylor drop radius $(\mathrm{m})$

Re Reynolds number

$t \quad$ Time (s)

$\vec{u} \quad$ Velocity vector $(\mathrm{m} / \mathrm{s})$

$V_{T} \quad$ Terminal velocity of Taylor drop $(\mathrm{m} / \mathrm{s}$

\section{Greek symbols}

a Volume fraction

$\kappa \quad$ Curvature $(\mathrm{m})$

$\lambda \quad$ Inelastic time constant (s)

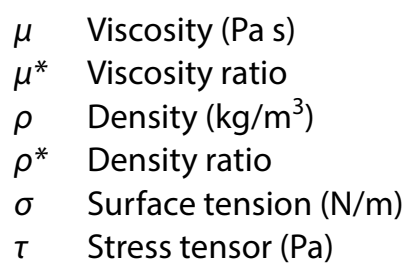

\section{Introduction}

The behavior and shape of the interface between phases in a multiphase flow lead to different flow patterns. In a multiphase flow, mechanisms and competitive forces determine the flow regime. One of the most important multiphase flow patterns is slug flow [1]. The slug flow is known as a flow pattern in which the dispersed phase is surrounded by a continuous phase in the form of large bubbles or drops (Taylor bubbles of Taylor drops). There

Morteza Bayareh, m.bayareh@sku.ac.ir | 'Department of Mechanical Engineering, Shahrekord University, Shahrekord, Iran. 
are many applications of slug flow in industries such as cooling in the nuclear reactors, heaters and fermentation vessels.

Many studies have considered the slug flow between gas and liquid phases. However, there is no a comprehensive knowledge about the liquid-liquid slug flow. Taylor and Davis [2] studied numerically and experimentally the motion of large bubbles in a liquid fluid. They carried out measurements related to the shape and ascending rate of air bubbles in nitrobenzene and water with different volumes and obtained bubble profiles. The large and long bubbles observed in this study were called Taylor bubbles. Ono and Kintner [3] investigated the rising motion of air bubbles in a quiescent liquid to determine their velocity for a wide range of fluid viscosity and surface tension in a vertical tube. They correlated the velocity of the rising bubble relative to the velocity of a similar moving sphere in the liquid using a correction coefficient. Maida [4] performed experiments on the rising motion of Taylor bubbles and spherical ones in stagnant and flowing fluids (water-air system) inside the vertical cylindrical tubes. He showed that in a stagnant fluid, there is a critical diameter for the tube that the bubble with smaller diameter than critical value cannot raise in the tube. Caro et al. [5] experimentally studied the motion of Taylor bubbles rising in Newtonian and shear-thinning non-Newtonian fluids in inclined tubes. They showed that an increase in the fluid viscosity or an increase in the surface tension causes the nose of the bubble to widen, and as a result, the bubble rise velocity decreases. Liao and Zhao [6] investigated a theoretical model to predict the velocity of a Taylor bubble in small vertical channels with square and triangle cross sections. The end of channels was blocked, and they were filled with a stagnant liquid. The corresponding relations were determined to predict bubble rise velocity in the channels using dimensionless parameters. Nogueira et al. [7] used PIV and shading techniques to study the effects of liquid viscosity on the flow and dynamical field of a rising Taylor bubble in stationary flows in a vertical tube. They found that the length of the Taylor bubble depends on the inverse of the viscosity. Luo and Prosperti [8] analyzed the linear stability of large gas bubbles that lost their stability during their rising motion. It was found that the relative velocity between the bubble and the liquid decreases with increasing the flow rate. Dirito et al. [9] studied numerically the motion of a Taylor drop in a vertical tube with a continuously heavier phase. Numerical simulations were carried out using a volume of fluid method for a symmetric geometry. They provided details of flow patterns and drop shapes under different conditions. The rising motion of a Taylor drop in stagnant liquids was investigated by Dirito et al. [10]

numerically. They reported drop velocity profile and demonstrated that axisymmetric behavior is not valid when viscosity ratio is very small or very large. Lertnuwat [11] proposed an empirical model to predict the shapes of a Taylor bubble rising in a stagnant liquid for different values of surface tension. It was concluded that since the surface tension impact on the Froude number, it controls the fluid flow around the Taylor bubble.

Present study aims to evaluate the motion of a Taylor drop in a non-Newtonian stagnant fluid in a vertical tube (Fig. 1). The main objective of the present work is to study the shear-thinning effect on the rising motion a Taylor drop. The geometry of the Taylor drop includes two hemispheres and a cylinder in such a way that the length of the tube is 8 times the length of the drop.

\section{Numerical method and governing equations}

The present study considers the motion of a Taylor drop rising in a liquid continues phase. There are some techniques to solve the governing equations such as level set (LS) $[12,13]$, coupled level set and volume of fluid (CLSVOF) [14], VOF [15-17] and front tracking techniques [18-24]. In the present study, to measure the motion of the two-phase flow, the VOF method is employed. To represent the fluids, the volume fraction of $a$ is used and defined as follows [16]:

Fig. 1 Geometry of the Taylor drop, $h=0.2 R$

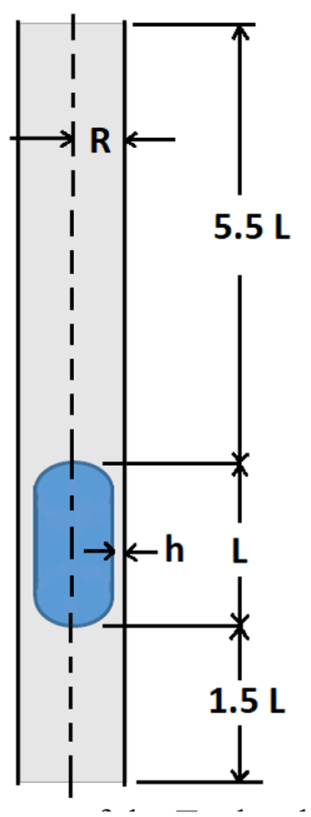


$\alpha=\frac{V_{1}}{V_{\text {cell }}}=\left\{\begin{array}{c}=1 \text { the cell is occupied by continuous phase } \\ 0<\alpha<1 \text { the cell includes the interface } \\ =0 \text { the cell is occupied by dispersed phase }\end{array}\right.$

The governing equations for unsteady, laminar and incompressible flow include continuity and Navier-Stokes equations $[16,23]$ :

$\nabla \cdot \vec{u}=0$,

$\frac{\partial}{\partial t}(\rho \vec{u})+\nabla \cdot(\rho \vec{u} \vec{u})=-\nabla p+\nabla \cdot \vec{\tau}+\rho \vec{g}+\sigma \kappa \nabla \alpha$

where $\vec{u}$ is the velocity vector, $p$ is the pressure, $\sigma$ is the surface tension, $\vec{g}$ is the gravity acceleration vector, and $k$ is the curvature. Due to the continuous surface force (CSF) at the interface of multicomponent fluid flow [25], the surface tension force is added to the Navier-Stokes equation as an additional source term $\sigma \kappa \nabla \alpha$. The curvature $\kappa$ is defined as the normal vector divergence:

$\kappa=-\nabla \cdot(\vec{n})$

In this study, glycerol-water solution is selected as continuous phase fluid. Glycerol-water solution is prepared by mixing certain weights of glycerol and distilled water. This non-Newtonian fluid is modeled by the Bird-Carreau fluid model. The Bird-Carreau model is defined as follows [23]:

$\vec{\tau}=\mu_{\dot{\gamma}} \dot{\gamma}$,

with

$\mu_{\dot{\gamma}}=\mu_{\infty}+\left(\mu_{0}-\mu_{\infty}\right)\left[1+(\lambda \dot{\gamma})^{a}\right]^{\frac{k-1}{2}}$

where $\mu_{0}$ is the zero shear rate viscosity, $\mu_{\infty}$ is an infinite shear rate viscosity, and $\lambda$ is the inelastic time constant. This fluid behaves as a Newtonian fluid in low shear rates $(\dot{\gamma} \ll 1 / \lambda)$ and behaves like power-law non-Newtonian fluid in high shear rates $(\dot{\gamma} \gg 1 / \lambda)$. The strain rate tensor $\dot{\gamma}$ is defined as [23]:

$\dot{\gamma}=(\nabla \vec{u})+(\nabla \vec{u})^{T}$

This non-Newtonian fluid is a generalized Newtonian fluid that its viscosity depends on the shear strain rate $\dot{\gamma}$.

Finite volume scheme is employed to discretize the governing equations. Also, PIMPLE algorithm is used for coupling the velocity and pressure fields [26]. This algorithm is a merged SIMPLE-PISO method.

The dimensionless governing parameters are as follows:

The density ratio is the ratio of the density of drop $\left(\rho_{d}\right)$ to the density of continuous phase $\left(\rho_{\mathrm{c}}\right)$ [18]:
Table 1 Physical properties of the glycerol-water solution and the Bird-Carreau model constants [27]

\begin{tabular}{lllll}
\hline$n$ & $\lambda(\mathrm{s})$ & $\mu_{0}(\mathrm{~Pa} \mathrm{~s})$ & $\mu_{\infty}(\mathrm{Pa} \mathrm{s})$ & $\rho\left(\mathrm{kg} / \mathrm{m}^{3}\right)$ \\
\hline 0.463 & 51 & 0.085 & 1.22 & 1220 \\
\hline
\end{tabular}

Table 2 Physical properties of silicon oil KF96-100 [27]

\begin{tabular}{ll}
\hline$\mu$ & $\rho\left(\mathrm{kg} / \mathrm{m}^{3}\right)$ \\
\hline 0.097 & 965 \\
\hline
\end{tabular}

$\rho^{*}=\frac{\rho_{\mathrm{d}}}{\rho_{\mathrm{c}}}$

The viscosity ratio is the ratio of the viscosity of drop $\left(\mu_{\mathrm{d}}\right)$ to the viscosity of continuous phase $\left(\mu_{\mathrm{c}}\right)$ [19]:

$\mu^{*}=\frac{\mu_{\mathrm{d}}}{\mu_{\mathrm{c}}}$

The Eötvös number [24]:

$\mathrm{Eo}_{D}=\frac{\Delta \rho g D^{2}}{\sigma}$

where $\Delta \rho=\rho_{\mathrm{c}}-\rho_{\mathrm{d}}$ and $D$ is the tube diameter.

Generalized Reynolds number [27]:

$\operatorname{Re}_{n}=\frac{\rho_{\mathrm{c}} V_{\mathrm{T}}^{2-n} D^{n}}{\mu_{\mathrm{c}} \lambda^{n-1}}$

where $V_{\mathrm{T}}$ is the terminal velocity of Taylor drop.

The Froude number [27]:

$\mathrm{Fr}=\frac{V_{\mathrm{T}}}{\sqrt{\Delta \rho g D / \rho_{\mathrm{c}}}}$

The physical properties of the glycerol-water solution and the Bird-Carreau model constants are presented in Table 1, and the physical properties of the Taylor drop (silicon oil KF96-100) are shown in Table 2.

\section{Results}

\subsection{Grid study}

To select the appropriate solution grid for the present simulations, the velocity of Taylor drop is calculated for several grid resolutions with 4704, 18,816 and 29,406 elements. Figure 2 shows the effect of the number of grid elements on the Taylor drop velocity. It is observed that the drop velocity does not change for the grid with more than 


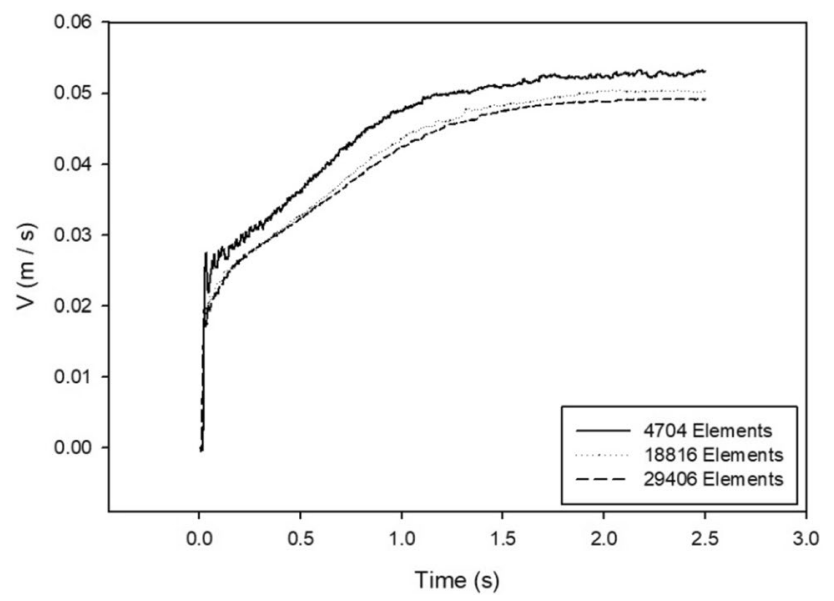

Fig. 2 Drop velocity versus time for different grid resolutions

18,816 elements. Hence, the grid resolution with 18,816 elements is employed for further simulations.

\subsection{Validation}

In order to validate the simulations, the present results are compared with the ones reported by Hayashi et al. [27] who considered the motion of a Taylor drop using the grid size of $\Delta r=\Delta z=R / 32$, where $R$ is the radius of hemisphere. KF96-100 silicon oil Taylor drop raises in a glycerol-water solution in a vertical tube with a circular cross sections ( 11 , 20,26 and $31 \mathrm{~mm}$ in diameter). The tube height is 18.375 times of the tube diameter, so that the number of cells in the network is $3776 \times 1176$. The density ratio is 0.79 , and surface tension between two phases is $0.031 \mathrm{~N} / \mathrm{m}$. Figure 3 shows the Froude number versus Eötvös number. According to the results, the maximum error of $0.4 \%$ is observed between the present results and those of Hayashi et al. [27].

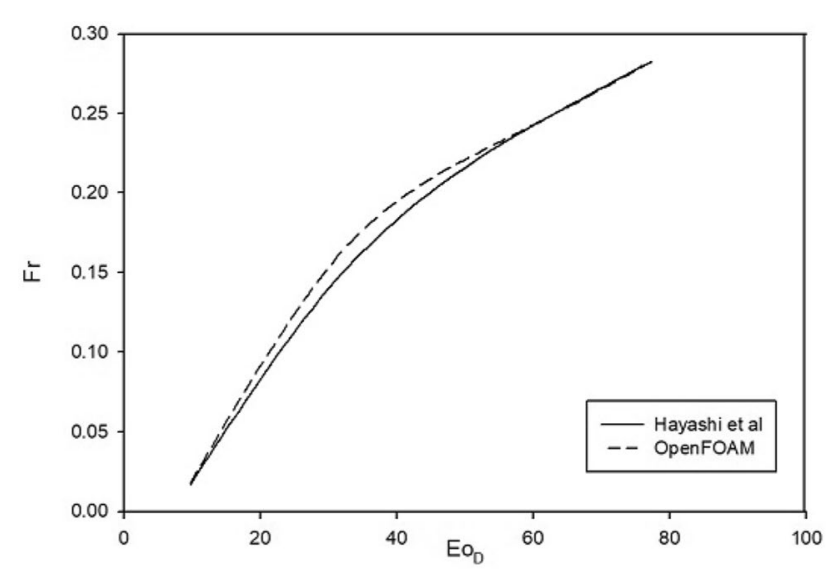

Fig. 3 Comparison of the present results and those of Hayashi et al. [27]
In the present study, the motion of a Taylor drop through a non-Newtonian fluid in a vertical tube is studied using open-source OpenFOAM software. Dimensionless parameters are limited to $9.5 \geq \mathrm{Eo}_{D} \geq 123.7$ and $0.05 \geq \mathrm{Fr} \geq 0.31$.

\subsection{Power-law index effect}

In the power-law Bird-Carreau model, $n=1, n<1$ and $n>1$ represent Newtonian, shear-thinning and shear-thickening fluids, respectively. In the present study, shear-thinning fluid $(n=0.2,0.463,0.6,0.8)$ is considered for continues phase. Non-Newtonian shear-thinning fluids are the fluids whose viscosity decreases with shear strain. This behavior is often seen in polymer solutions, molten polymers, compound fluids and suspensions, such as blood and color. In current simulations, the Eötvös number is 54.5. The force due to the viscosity leads to the dissipation of energy of the drop. By increasing the power-law index, the effect of the force due to the viscosity decreases and the kinetic energy of the drop increases. Therefore, the Froude number and the velocity of the Taylor drop increase. Figure 4 shows the increase in the Froude number with the powerlaw index of the fluid. The variation of the Froude number is such that, with the increase in the power-law index and the approach of the fluid behavior to the Newtonian fluid, the slope of the variation $\partial \mathrm{Fr} / \partial n$ increases. For very small power-law indices, changes in the power-law index do not have much effect on Froude number.

As shown in Fig. 5, Taylor drops will be larger and the thickness of the thin layer of the fluid will be less by increasing the power-law index. The reason is that the drop tends to occupy the entire width of the channel by increasing the power-law index and decreasing the viscosity. As the power-law index increases, dentures at the end

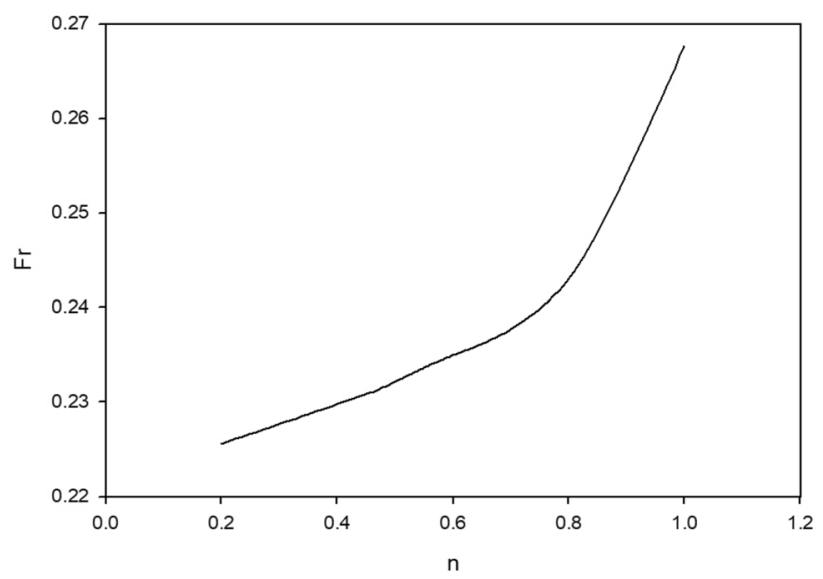

Fig. 4 Froude number as a function of power-law index for Eo $=54.5$ and $\rho^{*}=0.79$ 


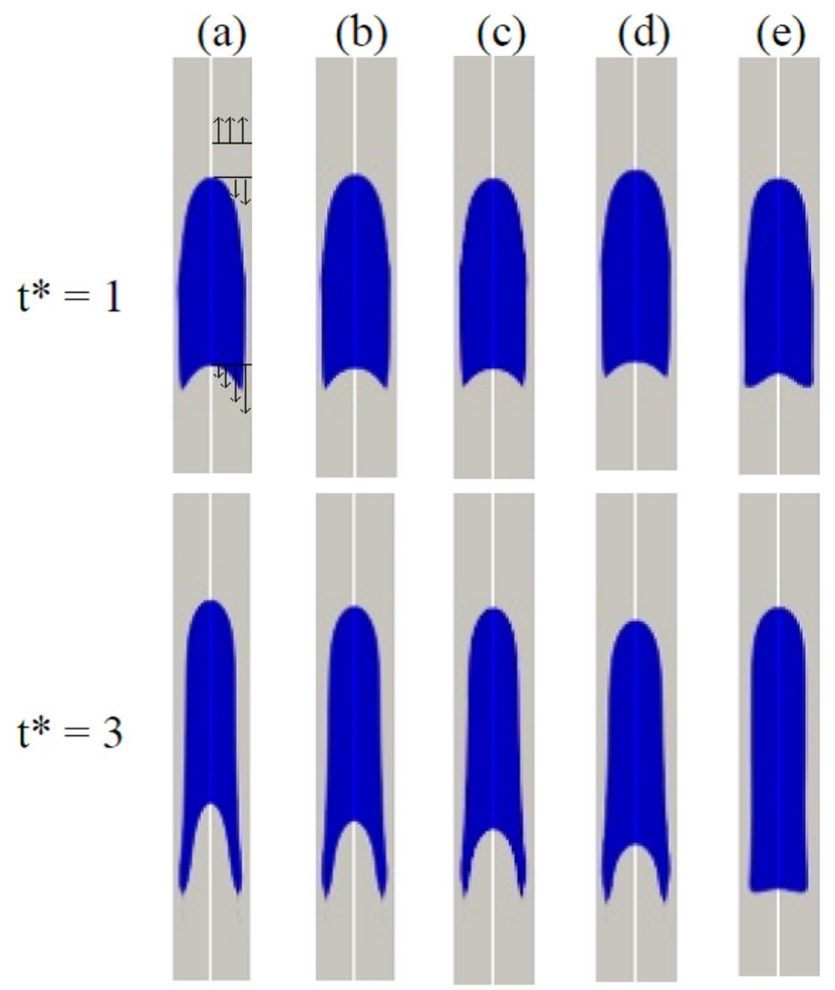

Fig. 5 Time evolution of a Taylor drop for different power-law indices and Eo $=54.5$ and $\rho^{*}=0.79$ : a $n=0.2$, b $n=0.463$, c $n=0.6$, $\mathbf{d} n=0.8$ and e $n=1$. First snapshot shows the variation of velocity profile along the channel

of the drop increase. As the power-law index increases, the drop velocity and the Froude number increase due to an increase in in the fluid viscosity. As the fluid viscosity increases, the stresses increase. The increase in these stresses leads to overcoming the surface tension force, which tends to maintain the cross section of the drop at its lowest value and creates a cavity at the end of the drop by applying stress concentration. For $n=1$, the base fluid behaves like Newtonian fluid and a cavity is not generated at the end of the drop. Hence, the Taylor drop is smaller compared to non-Newtonian cases.

Figure 6 shows that the drop velocity increases due to the loss of viscosity and the increase in the drop size. The slope of the variations of drop velocity is approximately the same for different power-law indices, and all drops reach their final velocity at $z / H=0.5$.

\subsection{Effect of the Eötvös number}

To investigate the effect of the surface tension on the motion and deformation of the Taylor drop, different Eötvös numbers are considered. It should be noted that the reduction in the Eötvös number means the overcoming of the force due to surface tension on the buoyancy force.

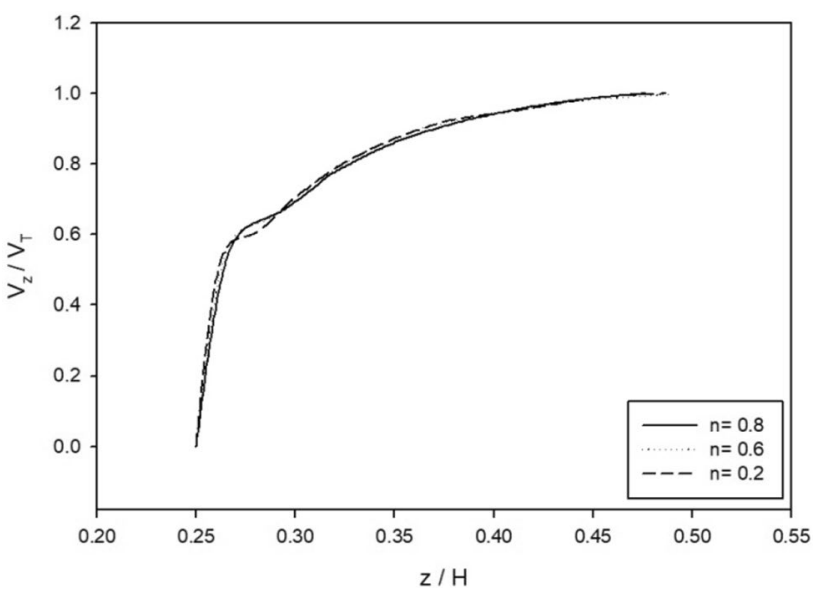

Fig. 6 Dimensionless velocity of a Taylor drop for different powerlaw indices, Eo $=54.5, \rho^{*}=0.79$ and $\log M=-2.5$

As the drop moves upward, after a while, the gravitational force causes the drop to pull downward and deviates from the spherical shape. Sphere has the minimum surface between all surfaces. When the surface tension force is dominant, it attempts to minimize the cross section of the drop, because this force always tries to keep the spherical shape of the drop and occupy the smallest possible area. According to Fig. 7, the drop becomes smaller and the tip of the drop becomes closer to the spherical shape with a decrease in the Eötvös number or an increase in the surface tension. Hence, the thickness of the thin layer of fluid that surrounds the drop is reduced by reducing the Eötvös number.

The effect of the surface tension force on the drop dynamics decreases by increasing the Eötvös number. The increase in the Eötvös number and size of the Taylor drop lead to an increase in its rising velocity. Figure 8 shows that the Froude number increases nonlinearly with the Eötvös number. The slope of the variation $\partial \mathrm{F}_{\mathrm{r}} / \partial \mathrm{Eo}_{D}$ decreases with the increase in the Eötvös number, so that for $\mathrm{Eo}_{D}>70$, the effect of the Eötvös number on the Froude number is negligible.

Figure 9 demonstrates that the slope of the velocity variation $\partial\left(V_{z} / V_{T}\right) / \partial(z / h)$ remains constant with the variation of the Eötvös number. It is revealed that the velocity of the drop is greater for the larger Eötvös numbers and the drop reaches its final velocity after rising less distance.

\section{Conclusions}

The present study investigated the motion of a Taylor drop in a non-Newtonian fluid in a vertical tube numerically. The governing equations were solved using VOF method in a symmetric geometry using OpenFOAM software. 


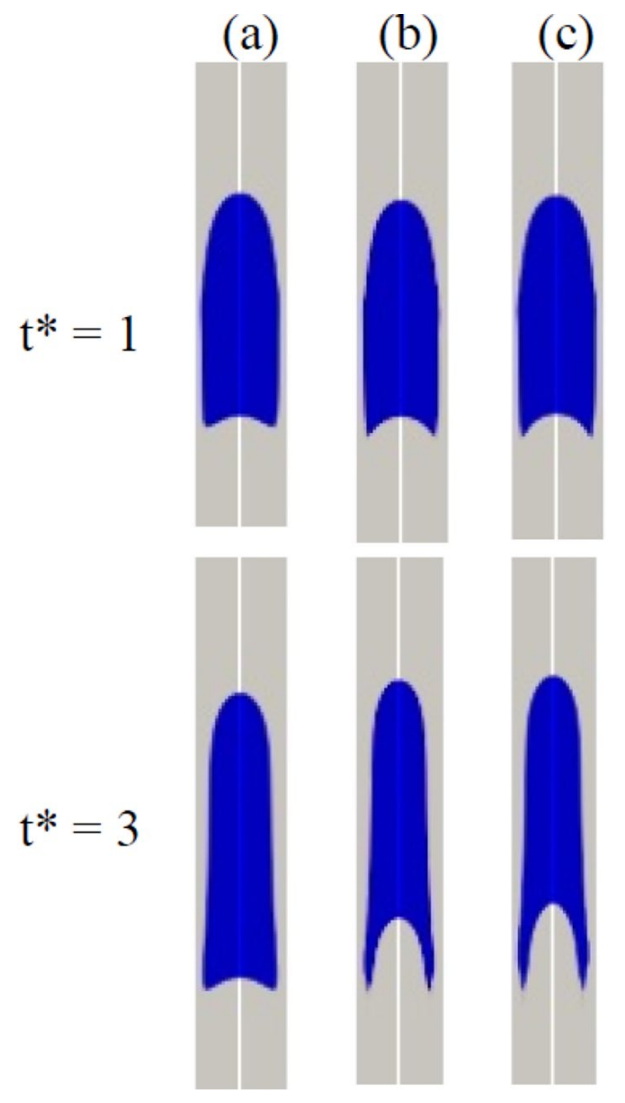

Fig. 7 Time evolution of a Taylor drop for different Eötvös numbers, $\rho^{*}=0.79$ and $n=0.463: \mathbf{a}$ Eo $=18.8, \mathbf{b}$ Eo $=54.5$ and $\mathbf{c}$ Eo $=84.6$

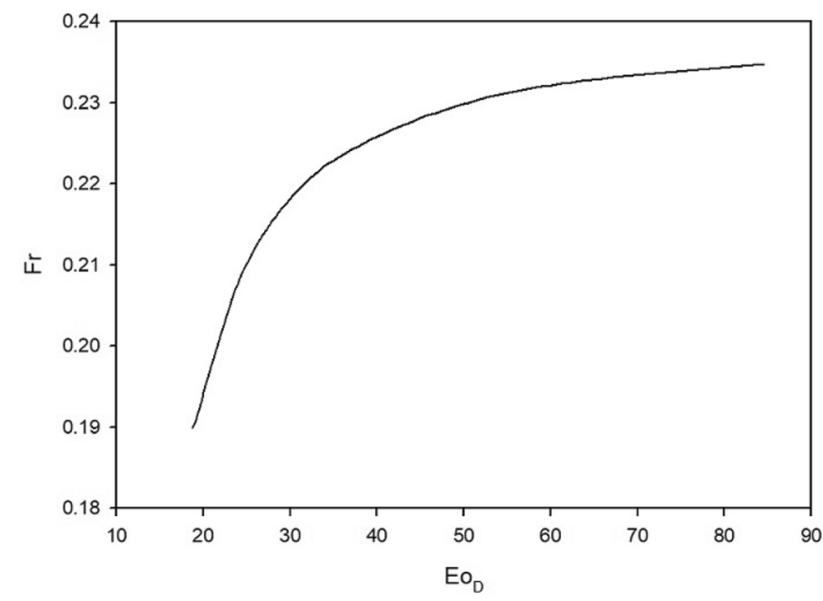

Fig. 8 Froude number versus Eötvös number for $\rho^{*}=0.79$ and $n=0.463$

The results demonstrated that the effect of the force due to the viscosity decreases and the kinetic energy of the drop increases by increasing the power-law index. Therefore, the Froude number and the velocity of the Taylor drop increase with $n$. It was revealed that the Taylor drop

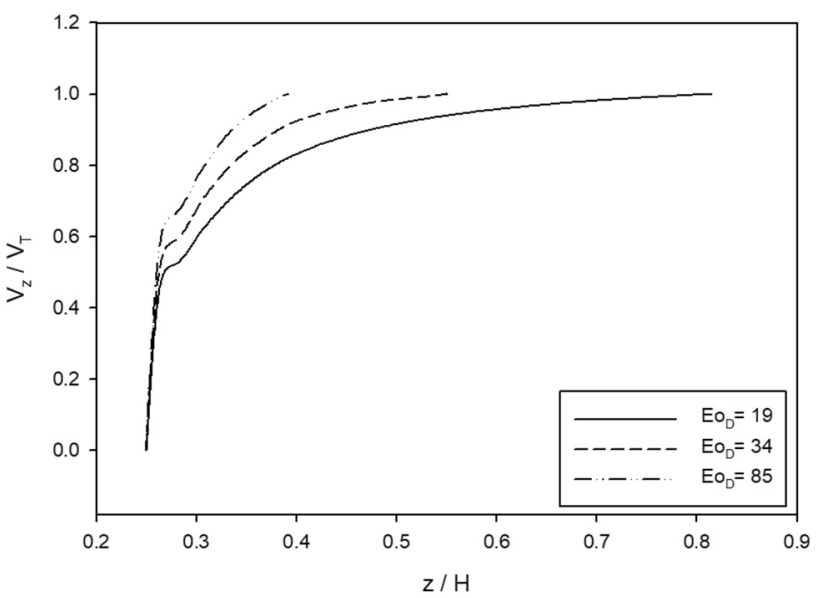

Fig. 9 Dimensionless velocity of a Taylor drop for different Eötvös numbers, $\rho^{*}=0.79$ and $n=0.463$

becomes smaller and the tip of the drop becomes closer to the spherical shape with a decrease in the Eötvös number or an increase in the surface tension. It was demonstrated that the drop deformation in Newtonian fluid $(n=1)$ is less than that in non-Newtonian ones. As the Eötvös number decreases, the thickness of the thin layer of the fluid and the drop deformation decrease. The results showed that for $\mathrm{Eo}_{D}>70$, the effect of the Eötvös number on the Froude number is negligible.

\section{Compliance with ethical standards}

Conflict of interest The authors declare that they have no conflict of interest.

\section{References}

1. Pinilla A, Guerrero E, Henao DH, Reyes DV, Pereyra E, Soto G, Ratkovich N (2019) CFD modelling of two-phase gas-liquid annular flow in terms of void fraction for vertical down- and up-ward flow. SN Appl Sci 1:1382. https://doi.org/10.1007/s4245 2-019-1430-3

2. Davies RM, Taylor SG (1950) The mechanics of large bubbles rising through extended liquids and through liquids in tubes. Proc R Soc Lond A 200:375-390. https://doi.org/10.1016/B978-0-08092523-3.50041-1

3. Uno S, Kintner RC (1956) Effect of wall proximity on the rate of rise of single air bubbles in a quiescent liquid. Am Inst Chem Eng 2(3):420-425. https://doi.org/10.1002/aic.690020323

4. Maeda M (1975) Behavior of a single bubble in a quiescent and following liquid inside a cylindrical tube. J Nucl Sci Technol 12(10):606-617. https://doi.org/10.1080/18811248.1975.97331 61

5. Carew PS, Thomas NH, Johnson AB (1995) A physically based correlation for the effects of power law rheology and inclination on slug bubble rise velocity. Int J Multiph Flow 21(6):1091-1106. https://doi.org/10.1016/0301-9322(95)00047-2

\section{SN Applied Sciences}


6. Liao Q, Zhao TS (2003) Modeling of Taylor bubble rising in a vertical mini noncircular channel filled with a stagnant liquid. Int J Multiph Flow 29:411-434. https://doi.org/10.1016/S0301 -9322(03)00004-1

7. Nogueira S, Rithmuiler ML, Pinto AMFR, Campos JBLM (2005) Flow in the nose region and annular film around a Taylor bubble rising through vertical columns of stagnant and flowing Newtonian liquids. Chem Eng Sci 61(2):845-857. https://doi. org/10.1016/j.ces.2005.07.038

8. Lu X, Prosperetti A (2006) Axial stability of Taylor bubbles. J Fluid Mech 568:173-192. https://doi.org/10.1017/S00221120060022 05

9. Direito FJN, Campos JBLM, Miranda JM (2017) A Taylor drop rising in a liquid co-current flow. Int J Multiph Flow 96:134-143. https://doi.org/10.1016/j.ijmultiphaseflow.2017.07.009

10. Direito FJN, Campos JBLM, Miranda JM (2016) Rising of a single Taylor drop in a stagnant liquid-2D laminar flow and axisymmetry limits. Phys Fluids 28(5):057101. https://doi. org/10.1063/1.4947426

11. Lertnuwat B (2018) Shapes of an air Taylor bubble in stagnant liquids influenced by different surface tensions. Int J Appl Mech Eng 23(1):79-90

12. Gu ZH, Wen HL, Yu CH, Sheu TWH (2018) Interface-preserving level set method for simulating dam-break flows. J Comput Phys $374: 249-280$

13. An RD, Yu CH (2019) A level set redistancing algorithm for simulation of two-phase flow. Fundam Numer Heat Transf B. https:// doi.org/10.1080/10407790.2020.1746601

14. Yu CH, Wen HL, Gu ZH, An RD (2019) Numerical simulation of dam-break flow impacting a stationary obstacle by a CLSVOF/ IB method. Commun Nonlinear Sci Numer Simul 79:104934

15. Gu ZH, Wen HL, Yao Y, Yu CH (2019) A volume of fluid method algorithm for simulation of surface tension dominant two-phase flows. Numer Heat Transf B: Fundam 76(1):1-17

16. Goodarzi Z, Ahmadi Nadooshan A, Bayareh M (2018) Numerical investigation of off-centre binary collision of droplets in a horizontal channel. J Braz Soc Mech Sci Eng 40:1-10. https:// doi.org/10.1007/s40430-018-1075-y

17. Hassanzadeh M, Ahmadi Nadooshan A, Bayareh M (2019) Numerical simulation of the head-on collision of two drops in a vertical channel. J Braz Soc Mech Sci Eng 41:1-14. https://doi. org/10.1007/s40430-019-1624-z
18. Bayareh M, Mortazavi S (2009) Geometry effects on the interaction of two equal-sized drops in simple shear flow at finite Reynolds numbers, 5th international conference: computational methods in multiphase flow. WIT Trans Eng Sci 63:379-388

19. Bayareh $M$, Mortazavi S (2013) Equilibrium position of a Buoyant drop in Couette and Poiseuille flows at finite Reynolds numbers. J Mech 20:53-58. https://doi.org/10.1017/jmech.2012.109

20. Bayareh $M$, Mortazavi S (2011) Binary collision of drops in simple shear flow at finite Reynolds numbers. Adv Eng Softw 42:604611. https://doi.org/10.1016/j.advengsoft.2011.04.010

21. Bayareh M, Mortazavi S (2011) Migration of a drop in simple shear flow at finite Reynolds numbers: size and viscosity ratio effects. In: Proceeding of international conference on mechanical, industrial, and manufacturing engineering (ICMIME)

22. Armandoost P, Bayareh M, Ahmadi Nadooshan A (2018) Study of the motion of a spheroidal drop in a linear shear flow. J Mech Sci Technol 32:2059-2067. https://doi.org/10.1007/s1220 6-018-0415-2

23. Mohammadi Masiri S, Bayareh M, Ahmadi Nadooshan A (2019) Pairwise interaction of drops in shear-thinning inelastic fluids. Korea-Austra Rheol J 31:25-34. https://doi.org/10.1007/s1336 7-019-0003-8

24. Bayareh M, Dabiri S, Ardekani AM (2016) Interaction between two drops ascending in a linearly stratified fluid. Eur J MechB/Fluids 60:127-136. https://doi.org/10.1016/j.euromechfl u.2016.07.002

25. Kim JS (2005) A continuous surface tension force formulation for diffuse-interface models. J Comput Phys 204:784-804

26. Issa R (1991) Solution of the implicitly discretised fluid flow equations by operator-splitting. J Comput Phys 93(2):388-410

27. Hayashi K, Kurimoto R, Tomiyama A (2011) Terminal velocity of a Taylor drop in a vertical pipe. Int J Multiph Flow 37:241-251. https://doi.org/10.1016/j.ijmultiphaseflow.2010.10.008

Publisher's Note Springer Nature remains neutral with regard to jurisdictional claims in published maps and institutional affiliations. 